\title{
Macrophage Polarization Modulates Development of Systemic Lupus Erythematosus
}

\author{
Feng Li Yongsheng Yang Xiaohua Zhu Lan Huang Jinhua Xu \\ Department of Dermatology, Affiliated Huashan Hospital of Fudan University, Shanghai, China
}

\section{Key Words}

Systemic lupus erythematosus (SLE) - Macrophage polarization - M1 macrophage - M2 macrophage $\cdot$ Clodronate $\cdot$ ALD-DNA - Adoptive transplantation

\begin{abstract}
Background/Aims: Macrophages have recently been shown to play a critical role in the pathogenesis of systemic lupus erythematosus (SLE). However, the underlying mechanisms remain unclear. Methods: Here, we used an activated lymphocyte-derived DNA (ALD-DNA) method to induce SLE in mice. We used a macrophage-specific eliminator clodronate to selectively deplete macrophages in mice. We isolated macrophages from bone marrow of the mice and used cytokines to differentiate $\mathrm{M} 1$ and $\mathrm{M} 2$ macrophages, respectively. Adoptive transplantation of M1 or M2 macrophages was performed in clodronate-treated mice. The effects on SLE were evaluated by serum anti-dsDNA autoantibody, by renal pathological changes, and by urine protein levels. Results: ALD-DNA induced SLE-like features in mice, manifested by induction of serum anti-dsDNA autoantibody, by renal pathological changes, and by increases in urine protein levels. Clodronate significantly decreased macrophages in mice, which significantly increased SLE severity. Adoptive transplantation of $M 2$, but not M1 macrophages significantly reduced SLE severity in clodronate- and ALD-DNA-treated mice. Conclusion: M1 and M2 macrophages play different roles in development of SLE. M1 macrophages increase the severity of SLE, while M2 macrophages reduce it. Modulation of macrophage polarity may be an attractive therapy for SLE.
\end{abstract}

Jinhua Xu

KARGER 125
Department of Dermatology, Affiliated Huashan Hospital of Fudan University,

12 Wulumuqizhong Road, Shanghai 200040 (China)

Tel. +862152887783, E-Mail xujinghua_hs@163.com 


\section{Introduction}

Systemic lupus erythematosus (SLE) is characterized by autoimmune disorders in multiple organs with autoantibody production [1-3]. In SLE, the immune tolerance is breached in that the immune system actively attacks autoantigens, resulting in autoantibody production and a vicious cycle of chronic inflammation and tissue damage [1-3]. Primary abnormalities of $\mathrm{B}$ and $\mathrm{T}$ lymphocytes have been considered to be major bases of SLE [1$3]$, while recent studies have shed light on the involvement of monocytes/macrophages in the pathogenesis of the disease [4-6], which acknowledges a role of innate immunity upon adaptive immunity in SLE.

Abnormalities in monocyte phenotype and function have been previously appreciated with a variety of autoimmune disorders, including SLE [4-6]. The definition of macrophage activation mostly commonly uses terms of M1 and M2 macrophages, based on differential effects of interleukin-4 (IL-4) and interferon gamma (IFN- $\gamma$ ) on macrophage gene expression [7-11]. This definition originated from the differential metabolism of arginine between macrophages from C57BL/6 and macrophages from Balb/c mice, an effect he correlated with differences between T helper 1 (Th1) and Th2 cell responses in the same strains [12, 13]. Indeed, M1 macrophages respond to Th1 and generate reactive oxygen species and nitric oxide to kill pathogens and cells, while M2 macrophages respond to Th2 to mediate humoral immunity and tissue repair [7-13]. The differential activation of macrophage phenotypes is also called polarization $[12,13]$. In vitro, bone marrow derived macrophages are often polarized to M1 or M2 macrophages by treated with either IFN- $\gamma$ or IL-4 $[12,13]$.

Liposome-encapsulated clodronate is a first generation bisphosphonate drug encapsulated in a liposome for depletion of macrophages in the body [14-16]. There is a large body of evidence in mice indicating that systemic administration of clodronate efficiently depletes macrophages in a variety of organs and tissue [14-16]. Clodronate cannot ender the cells itself, and it needs liposome as a carrier to introduce clodronate into the cytoplasm of macrophages, taking advantage of the phagocytosis property of macrophages [14-16]. Intracellular macrophages induce rapid onset of apoptosis via competition for ATP binding to cellular substrates [14-16]. Moreover, some studies have indicated that systemic clodronate administration can induce significant antitumor activity based on tumorassociated macrophage depletion [17-21]. Depletion of macrophages by clodronate has also been used for control of inflammation [11,22-24], and in some autoimmune diseases, while its effects on these autoimmune diseases may be disease-dependent.

Here, we used an activated lymphocyte-derived DNA (ALD-DNA) method to induce SLE in mice [25-27]. We used clodronate to selectively deplete macrophages in these mice. We isolated macrophages from bone marrow of the mice and used cytokines to differentiate M1 and M2 macrophages, respectively. Adoptive transplantation of M1 or M2 macrophages was performed in clodronate-treated mice. The effects on SLE were evaluated by serum anti-dsDNA autoantibody, by renal pathological changes, and by urine protein levels. We found that ALD-DNA induced SLE-like features in mice. Clodronate significantly decreased macrophages in mice, which significantly increased SLE severity. Adoptive transplantation of M2, but not M1 macrophages significantly reduced SLE severity in clodronate- and ALDDNA-treated mice.

\section{Materials and Methods}

Mouse handling

All animal experiments were performed according to the Guide for the Care and Use of Medical Laboratory Animals (Ministry of Health, China, 1998) and the guidelines of the Fudan University Medical Laboratory Animal Care and Use Committee. Ten-week-old female Balb/c mice (Jackson lab, Bar Harbor, ME, USA) were housed in the pathogen-free mouse colony at our institution, and were used for the current study.

\section{KARGER}




\section{Cellular Physiology Cell Physiol Biochem 2015;37:1279-1288 \begin{tabular}{ll|l} 
and Biochemistry & $\begin{array}{l}\text { DOI: 10.1159/000430251 } \\
\text { Published online: October 05, 2015 }\end{array}$ & $\begin{array}{l}\text { (c) 2015 S. Karger AG, Basel } \\
\text { www.karger.com/cpb }\end{array}$ \\
\hline
\end{tabular}}

Li et al.: Macrophage Polarization in SLE

DNA preparation

Genomic DNAs from syngeneic Con A-activated or unactivated splenocytes were extracted and treated with S1 nuclease (Sigma-Aldrich, St. Louis, MO, USA) and proteinase K (Sigma-Aldrich) according to the manufacturer's instructions, and then purified using the UltraPureTM genomic DNA purification kit, as recommended by the manufacturer (Shanghai SBS Genetech, China). DNA concentrations were determined by absorbance measurement at $260 \mathrm{~nm}$. The final A260/A280 for all the DNA preparations was >1.8.

\section{Animal immunization}

BALB/c mice were divided into several groups of 10 mice and actively immunized by subcutaneous injection on the back with $0.2 \mathrm{ml}$ of an emulsion containing the indicated doses of ALD-DNA or Un-ALD-DNA in phosphate-buffered saline (PBS). Mouse blood was taken from retro-orbital sinus prior to immunization and at 2-week internals until the end of the experiment.

ELISA

Anti-ssDNA and anti-dsDNA Ab titers were assessed using an enzyme-linked immunosorbent assay (ELISA). Briefly, 96-well protamine sulphatepretreated polystyrene microtitre plates were coated with prepared DNA at $50 \mu \mathrm{g} / \mathrm{ml}$ and placed overnight at $4^{\circ} \mathrm{C}$ after incubation at $37^{\circ} \mathrm{C}$ for $2 \mathrm{~h}$. The plates were then washed three times with PBS containing 0.05\% Tween-20 (PBST). HRP-conjugated goat anti-mouse polyvalent (IgG, IgM, IgA) Ab (Sigma-Aldrich) was used as the secondary Ab. The absorbance was measured at $490 \mathrm{~nm}$ on a microtiter plate reader (R\&D System, Los Angeles, CA, USA). Results are presented as the mean of triplicate determinations on sera from individual mice.

Isolation, culture and differentiation of bone-marrow derived mouse macrophages

Isolation of bone-marrow derived mouse macrophages has been described before [28]. Marrow from female, 10 week-old Balb/c mice was flushed out with vehicle solution (PBS containing 20mmol/l Tris and $100 \mathrm{mmol} / \mathrm{l} \mathrm{NaCl}, \mathrm{pH} 7.5$ ) through a $23 \mathrm{G}$ needle. Cells were pre-treated with FITC-conjugated F4/80 antibody (Becton-Dickinson Biosciences, San Jose, CA, USA) and then sorted for positive cells by flow cytometry. Purified F4/80-positive macrophages were cultured in Dulbecco's Modified Eagle Medium/F12 (DMEM/ F12; Invitrogen, St. Louis, MO, USA) supplemented with $10 \mathrm{mmol} / \mathrm{l}$ L-glutamine, $100 \mathrm{U} / \mathrm{ml}$ penicillin, $100 \mu \mathrm{g} /$ $\mathrm{ml}$ streptomycin and $100 \mathrm{U} / \mathrm{ml}$ recombinant M-CSF (mouse M-CSF, R\&D Systems). In vitro differentiation of macrophages have been performed as has been previously described [28, 29].

\section{Fluorescence-activated cell sorting (FACS) for macrophage (subtype)}

Treated bone-marrow derived cells were detached from the culture plates with $0.25 \%$ Trypsine solution (Invitrogen), washed three times with PBS, re-suspended, labeled with FITC-conjugated F4/80 antibody and/or APC-conjugated MGL antibody (Becton-Dickinson Biosciences), and then sorted for macrophages, or M1 and M2 subtypes. Data were analyzed and quantified using Flowjo software (Flowjo LLC, Ashland, OR, USA).

\section{Clodronate injection}

Intravenous tail vein injection of $200 \mu \mathrm{l}$ clodronate-liposomes (Clodronateliposome, Amsterdam, Netherland) every week was performed as has been described before, to eliminate macrophages. Control mice received injections of liposome of the same amount and volume. For the adoptive M1 or M2 macrophage transplantation, macrophages were transplanted via tail vein to the circulation.

Assessment of stage score for lupus nephritis

Glomeruli in each mouse were scored blindly by an experienced pathologist for the stage score for lupus nephritis as following. Normal appearance: score 0; Class I (mild disease with small amount of swelling): score 1; Class II (still fairly mild disease but more swelling than Class I): score 2; Class III (moderate degree of swelling with less than $50 \%$ of the filtering glomeruli affected): score 3; Class IV (severe degree of swelling with greater than 50\% filtering glomeruli affected): score 4; Class V (most of the swelling is confined to the outer layer surrounding the filter unit): score 5; Class VI (most of the filter glomeruli show scarring): score 6. In each mouse, 100 glomeruli were assessed.

\section{KARGER}




\section{Cellular Physiology Cell Physiol Biochem 2015;37:1279-1288 \begin{tabular}{l|l|l}
\hline DOI: 10.1159/000430251 & (C) 2015 S. Karger AG, Basel
\end{tabular} www.karger.com/cpb

\section{Assessment of proteinuria}

Proteinuria was measured with the Coomassie brilliant blue assay. Albumin (bovine serum, SigmaAldrich) was used as standard curves. Murine urine were centrifuged ( $20 \mathrm{~min}, 4500 \mathrm{rpm}$ ), and supernatants were taken and diluted at 1:10 with normal saline. After Coomassie brilliant blue solution were added, the sample were tested at $540 \mathrm{~nm}$ and the concentration of proteinuria could be calculated by standard curves.

\section{$R T-q P C R$}

RNA was extracted from purified macrophages after FACS with RNeasy (Qiagen, Hilden, Germany). Complementary DNA (cDNA) synthesis was performed by reserve transcription. Quantitative PCR (RTqPCR) was performed in duplicates with QuantiTect SYBR Green PCR Kit (Qiagen). All primers were purchased from Qiagen. Quantification used ddCT method. Values of genes were first normalized against $\beta$-actin, and then compared to controls.

\section{Statistics}

GraphPad Prism software (GraphPad Software, Inc. La Jolla, CA, USA) was used for statistical analyses. Unpaired two-tailed Student t test was applied for comparison between two groups, and one-way ANOVA with the Tukey posttest was applied for comparison between three or more groups. Data were represented as mean \pm SD and were considered significant if $\mathrm{p}<0.05$.

\section{Results}

Systemic administration of clodronate significantly reduced monocytes/macrophages in circulation and in organs in mice

First, we examined whether tail vein injection of clodronate may substantially eliminate macrophages. We injected clodronate-liposome (clodronate) or control liposome into the mice and examined the macrophages in spleen after one week. We found that the spleen from the mice that received clodronate contained significantly less F4/80+ macrophages,

Fig. 1. Systemic administration of clodronate significantly reduced monocytes/ macrophages in circulation and in organs in mice. (A-C) We injected clodronate-liposome (clodronate) or control liposome into the mice and examined the macrophages in spleen after one week. We found that the spleen from the mice that received clodronate contained significantly less F4/80+ macrophages, shown by representa-

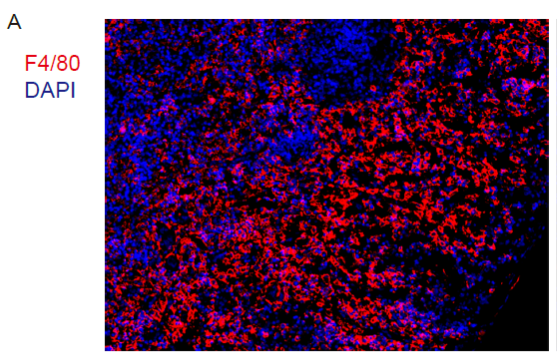

Liposome

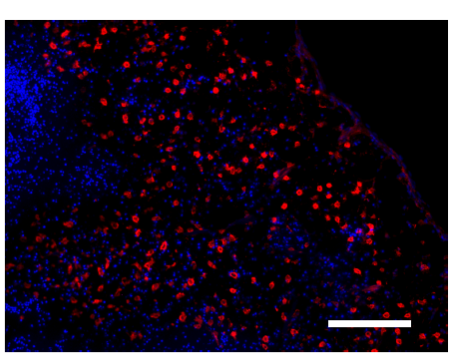

Clodronate-liposome
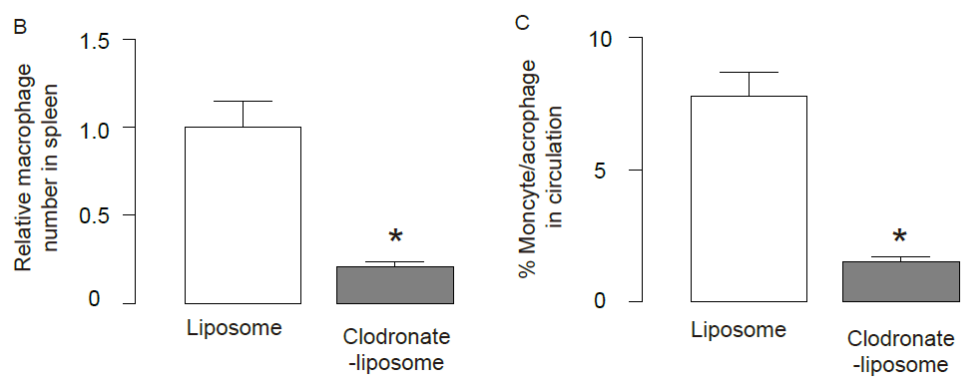

tive immunostaining images (A), and by quantification (B). (C) We also measured the percentage of monocytes/macrophages in the circulation one week after clodronate administration, and found that clodronate significantly decreased the percentage of monocytes/macrophages in the circulation. F4/80 in red, DAPI in blue. Scale bar is $50 \mu \mathrm{m} .{ }^{*} \mathrm{p}<0.05 . \mathrm{N}=10$. Statistics: one-way ANOVA with the Tukey posttest. 


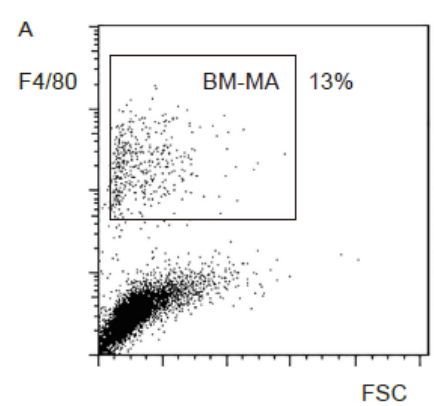

C

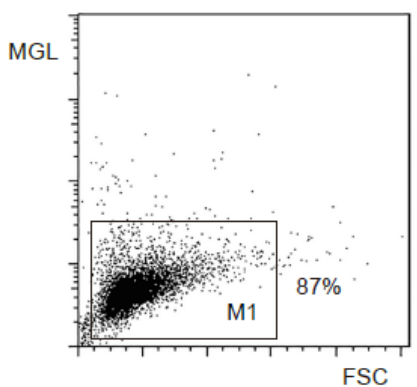

B

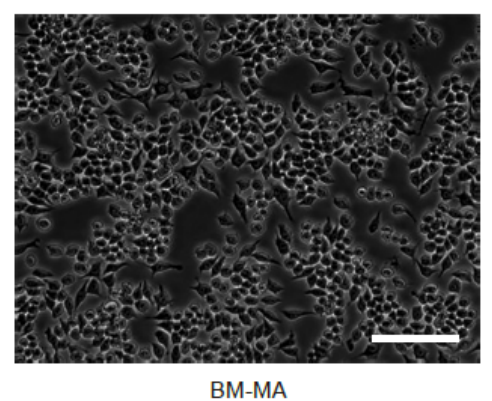

D

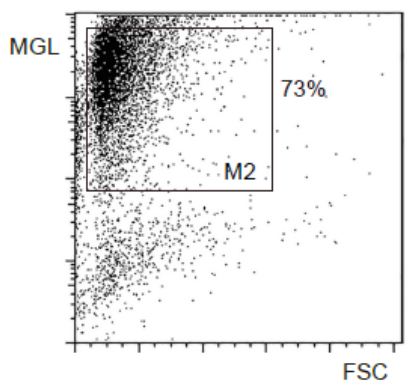

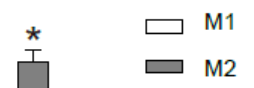

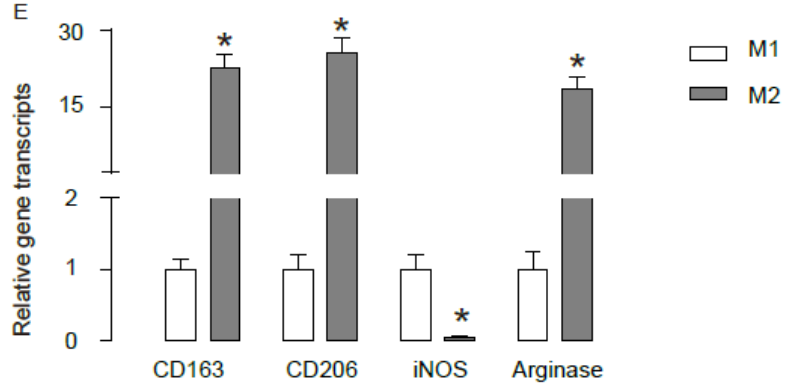

Fig. 2. Isolation and differentiation of bone-marrow derived macrophages. (A-B) We isolated mouse macrophages from bone marrow based on F4/80 expression by flow cytometry (A) and then put these macrophages in culture (B). (C-D) Macrophages were treated with either IFN- $\gamma$ (C), or IL-4 (D), and analyze and purify M1 and M2 activation, respectively, by flow cytometry. The purity of isolated M1 or M2 macrophages was examined by RT-qPCR for CD163, CD206, iNOS and Arginase. BM-MA: bone-marrow derived macrophages. FSC: forward scatter. Scale bar is $50 \mu \mathrm{m} .{ }^{*} \mathrm{p}<0.05$. N=10. Statistics: one-way ANOVA with the Tukey posttest.

shown by representative immunostaining images (Fig. 1A), and by quantification (Fig. 1B). We also measured the percentage of monocytes/macrophages in the circulation one week after clodronate administration, and found that clodronate significantly decreased the percentage of monocytes/macrophages in the circulation (Fig. 1C). These data suggest that systemic administration of clodronate significantly reduces monocytes/macrophages in circulation and in organs in mice.

\section{Isolation and differentiation of bone-marrow derived macrophages}

Next, we examined whether M1 and M2 macrophages could be prepared in vitro. We isolated mouse macrophages from bone marrow based on F4/80 expression by flow cytometry (Fig. 2A) and then put these macrophages in culture (Fig. 2B). Macrophages were treated with either IFN- $\gamma$, or IL-4, to induce M1 and M2 activation, respectively. The polarized activation of M1 (Fig. 2C) and M2 (Fig. 2D) macrophages was confirmed by differential expression of a M2-specific marker MGL on the cells by flow cytometry. M1 or M2 macrophages were isolated and further confirmed of the phenotype by RT-qPCR 

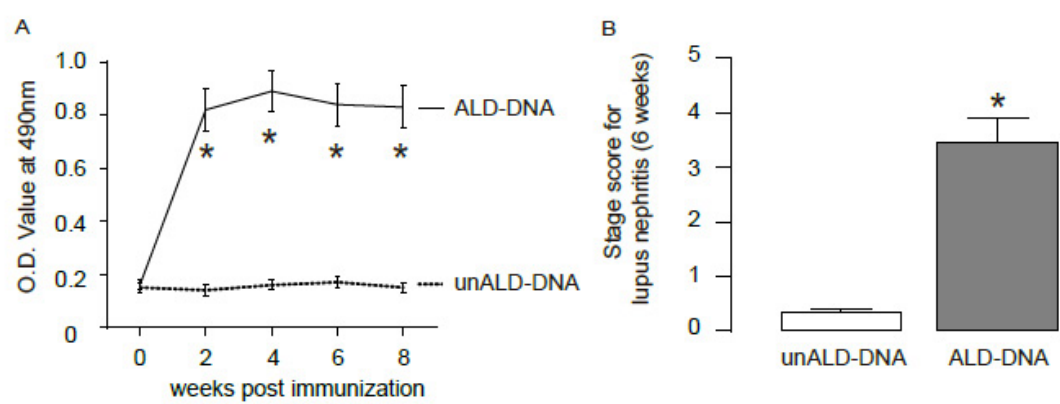

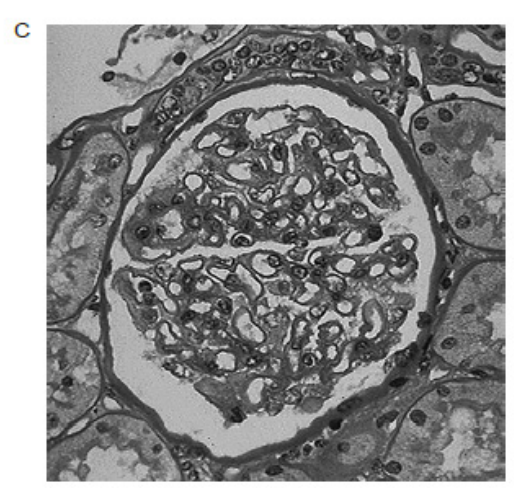

unALD-DNA

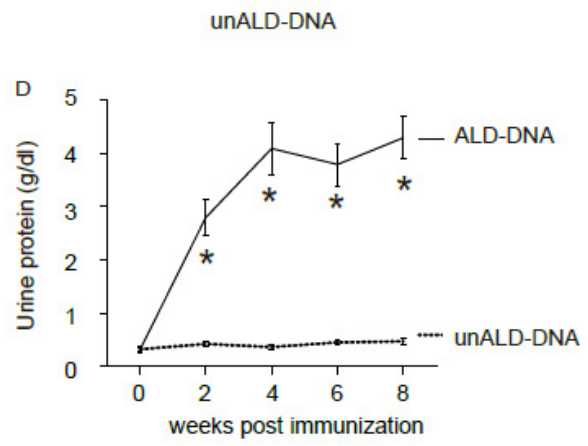

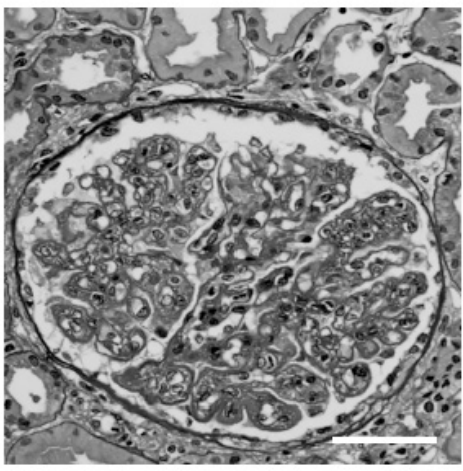

ALD-DNA

Fig. 3. Induction of SLE-like model in mice. (A) Groups of $10 \mathrm{BALB} / \mathrm{c}$ mice were first administered $50 \mu \mathrm{g}$ of ALD DNA or UnALD DNA. The levels of anti-dsDNA antibodies in the mouse sera were examined. We detected significantly higher levels of IgG anti-dsDNA antibodies in the sera from ALD-DNA-immunized mice, compared to unALD-DNA-immunized mice. (B-C) The pathological changes in kidneys were then assessed, showing significantly higher score for lupus nephritis in ALD-DNA-immunized mice, compared to unALD-DNA-immunized mice, by quantification (B), and by representative histological images (C). (D) ALD-DNA-immunized mice had significantly higher urine protein, compared to unALD-DNA-immunized mice. ${ }^{*} \mathrm{p}<0.05 . \mathrm{N}=10$. Statistics: one-way ANOVA with the Tukey posttest.

examination of CD163, CD206 and Arginase, which are predominantly expression by M2 macropahges, and of iNOS, which is predominantly expressed by M1 macrophages (Fig. 2E). Thus, M1 and M2 macrophages that were prepared in vitro are proper to be used in the adoptive transplantation.

\section{Induction of SLE-like model in mice}

Next, we used a previously established SLE model, in which anti-dsDNA antibodies were induced by immunization with ALD DNA. Thus, groups of 10 BALB/c mice were first administered $50 \mu \mathrm{g}$ of ALD DNA or UnALD DNA. First, this model was validated by examination 


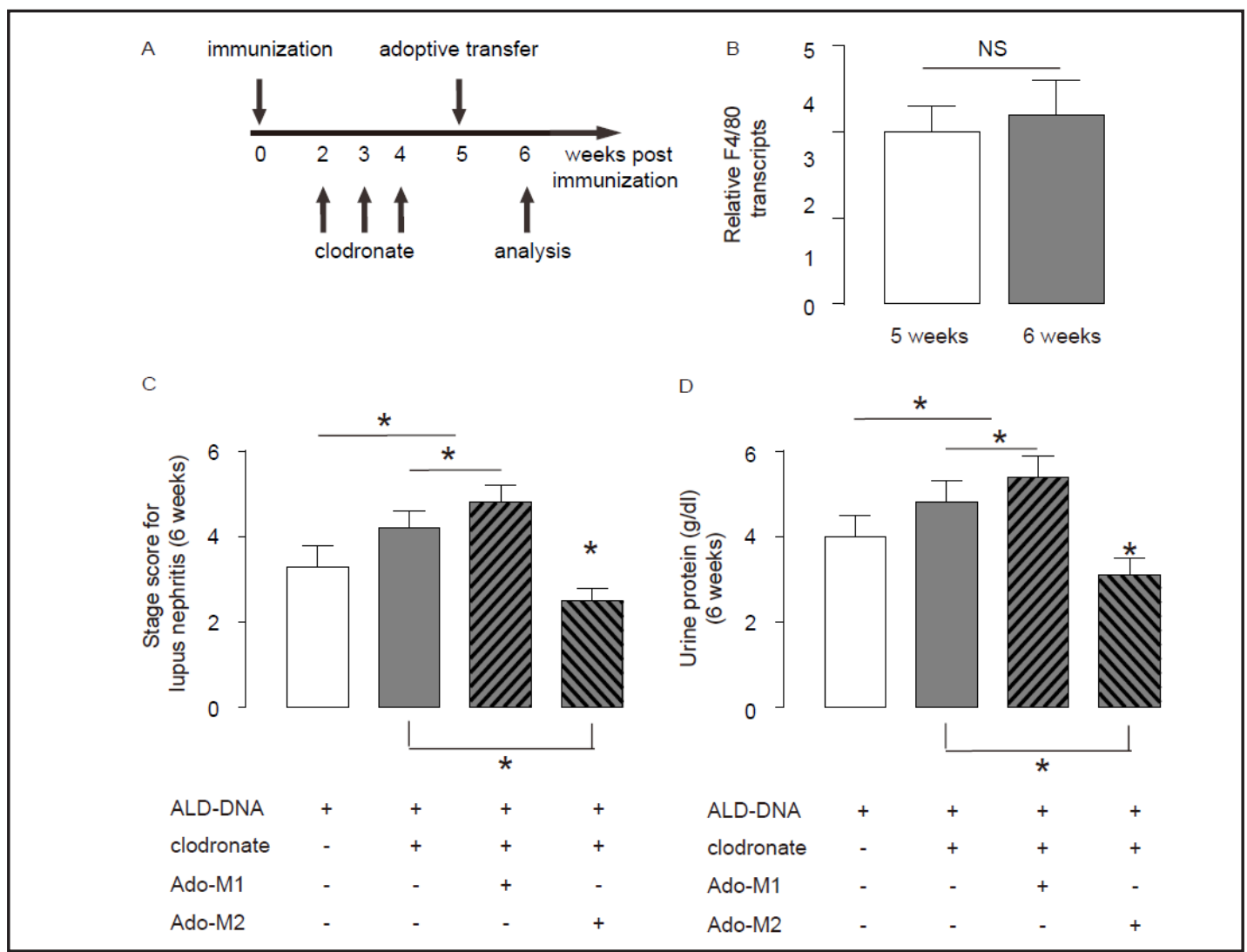

Fig. 4. Adoptive transplantation of M2 macrophages, but not M1 macrophages, abolished clodronate-induced augment in SLE severity. (A) Clodronate was given at 2, 3, 4 weeks after immunization to eliminate macrophages, and adoptive transplantation of either M1 macrophages or M2 macrophages in clodronate-treated, ALD-DNA-administrated mice was performed at 5 weeks. Analyses were performed at 6 weeks. (B) One week is sufficient for systemic elimination of Clodronate. (C) Clodronate significantly increased the SLE-associated lupus nephritis score, which was abolished by adoptive transplantation of M2 macrophages, but not M1 macrophages. (D) Clodronate significantly increased the urine protein, which was abolished by adoptive transplantation of M2 macrophages, but not M1 macrophages. ${ }^{*} \mathrm{p}<0.05$. NS: non-significant. $\mathrm{N}=10$. Statistics: one-way ANOVA with the Tukey posttest.

of the levels of anti-dsDNA antibodies in the mouse sera. We detected significantly higher levels of IgG anti-dsDNA antibodies in the sera from ALD-DNA-immunized mice, compared to unALD-DNA-immunized mice (Fig. 3A). The induction of anti-dsDNA antibodies was detected as early as 2 weeks after immunization, and appeared to be sustained for no less than 8 weeks (Fig. 3A). The pathological changes in kidneys were then assessed, showing significantly higher score for lupus nephritis in ALD-DNA-immunized mice, compared to unALD-DNA-immunized mice (Fig. 3B-C). Moreover, ALD-DNA-immunized mice had significantly higher urine protein, compared to unALD-DNA-immunized mice (Fig. 3D). Together, these hallmark features for SLE suggest that ALD-DNA indeed induces a SLE-like model in mice.

\section{Elimination of macrophage population by clodronate aggravated SLE severity}

Then, we used this model to study the role of macrophage/macrophage subtypes in SLE development. Clodronate was given at 2, 3, 4 weeks after immunization to eliminate macrophages, and analyses were performed at 6 weeks (Fig. 4A). We found that clodronate significantly increased the SLE-associated lupus nephritis score (Fig. 4B), and the urine 
protein levels (Fig. 4C). These data suggest that macrophages may have a protective role against SLE-associated autoimmunity.

Adoptive transplantation of M2 macrophages, but not M1 macrophages, abolished clodronate-induced augment in SLE severity

In order to figure out the exact roles of macrophage subtypes in the development of SLE, we did adoptive transplantation of either M1 macrophages or M2 macrophages in clodronatetreated, ALD-DNA-administrated mice at 5 weeks (Fig. 4A). Clodronate at 2, 3, 4 weeks combined with adoptive transplantation of M1 macrophages at 5 weeks mimicked the effects of selective elimination of M2 macrophages, while clodronate at 2, 3, 4 weeks combined with adoptive transplantation of M2 macrophages at 5 weeks mimicked the effects of selective elimination of M1 macrophages. Adoptively transplanted macrophages were not eliminated one week after termination of clodronate treatment (Fig. 4B), suggesting that clodronate could be systemically eliminated within one week. We found that adoptive transplantation of M2 macrophages, but not M1 macrophages, abolished clodronate-induced augment in SLE severity, shown by SLE-associated lupus nephritis score (Fig. 4C), and by the urine protein levels (Fig. 4D). These data suggest that M1 and M2 macrophages play different roles in development of SLE. M1 macrophages increase the severity of SLE, while M2 macrophages reduce it. Modulation of macrophage polarity may be an attractive therapy for SLE.

\section{Discussion}

Patients with SLE have evidence of dysfunction of monocyte/Macrophages, as a phenomenon that correlates with disease activity [4-6]. The macrophage activation by IL-4 or IFN- $\gamma$ results in different polarization of macrophages into M2, or M1 subtype [7-13]. These two types of macrophages play distinct roles in various physiological and pathological events, and often antagonize the activities and functions of each other [7-13]. Nevertheless, macrophage polarization in SLE pathogenesis is just recently appreciated but the underlying mechanisms remain largely unexplored.

Here, we address this question using a well-established SLE model, in which antidsDNA antibodies were induced by immunization with ALD DNA. To evaluate the roles of macrophages/macrophage subtypes in SLE, we used a specific macrophage eliminator clodronate to deplete all macrophages. Adoptive transplantation of in-vitro differentiated M1 or M2 macrophages in clodronate-treated mice was used as gain-of-function and loss-offunction experiments [7-13]. M1 and M2 macrophages were discriminated by MGL, which is a specific M2 macrophage marker like CD163 or CD206. First, we validated all these models. The SLE mouse model was confirmed by hallmark features for SLE, including induction of anti-dsDNA autoantibodies, development of lupus nephritis and protein urination [25-27]. The effects of systemic administration of clodronate on monocytes/macrophage depletion were confirmed by quantification of the monocytes/macrophages in circulation and in spleen as a representative organ [14-16]. The in-vitro differentiated bone-marrow-derived M1 or M2 macrophages were quality controlled by examination of M2-specific markers CD163, CD206 and Arginase, and of M1-specific marker iNOS [14-16].

Then, we used this validated model to study the role of macrophage/macrophage subtypes in SLE development. Clodronate was given at 2, 3, 4 weeks after immunization to eliminate macrophages, while adoptive transplantation of M1 or M2 macrophages was performed at 5 weeks. The one-week interval between last clodronate injection and macrophage adoptive transfer allows the systemic excretion of clodronate to be finished [14-16], and this was confirmed in our study. Thus, the transplanted macrophages will not be affected. We found that clodronate significantly aggravated the SLE-associated lupus nephritis and proteinuria, while adoptive transplantation of M2 macrophages, but not M1 macrophages, abolished clodronate-induced augment in SLE severity. These data suggest that M1 and M2 macrophages play different roles in development of SLE. M1 macrophages 
appear to increase the severity of SLE, while M2 macrophages reduce it. The net effect may be determined by the relative ratio of M1 vs M2 macrophages. Thus, modulation of macrophage polarity, specifically by induction of M2 macrophages may be an attractive therapy for SLE. Since IL-4 is a potential inducer for M2 macrophages, our data suggest that M2-inductive cytokines like IL-4 may have a therapeutic effects to alleviate SLE symptoms. Future experiments may address the molecular mechanisms underlying the regulation of different macrophage subtypes on SLE pathogenesis, and on M2-macrophage-associated therapy.

\section{Acknowledgement}

This work was supported by Shanghai Clinical Subject Construction Award (NO: AB83070002012016).

\section{Disclosure Statement}

The authors have declared that no competing interests exist.

\section{Reference}

1 Agmon-Levin N, Blank M, Paz Z, Shoenfeld Y: Molecular mimicry in systemic lupus erythematosus. Lupus 2009;18:1181-1185.

2 Crispin JC, Tsokos GC: Novel molecular targets in the treatment of systemic lupus erythematosus. Autoimmun Rev 2008;7:256-261.

3 Crispin JC, Kyttaris V, Juang YT, Tsokos GC: Systemic lupus erythematosus: New molecular targets. Ann Rheum Dis 2007;66:Suppl iii65-69.

4 Vilaiyuk S, Sirachainan N, Wanitkun S, Pirojsakul K, Vaewpanich J: Recurrent macrophage activation syndrome as the primary manifestation in systemic lupus erythematosus and the benefit of serial ferritin measurements: A case-based review. Clin Rheumatol 2013;32:899-904.

$5 \quad$ Orme J, Mohan C: Macrophage subpopulations in systemic lupus erythematosus. Discov Med 2012;13:151158.

$6 \quad$ Li Y, Lee PY, Reeves WH: Monocyte and macrophage abnormalities in systemic lupus erythematosus. Arch Immunol Ther Exp (Warsz) 2010;58:355-364.

7 Gordon S: Alternative activation of macrophages. Nat Rev Immunol 2003;3:23-35.

8 Gordon S, Martinez FO: Alternative activation of macrophages: Mechanism and functions. Immunity 2010;32:593-604.

9 Geissmann F, Manz MG, Jung S, Sieweke MH, Merad M, Ley K: Development of monocytes, macrophages, and dendritic cells. Science 2010;327:656-661.

10 Ricardo SD, van Goor H, Eddy AA: Macrophage diversity in renal injury and repair. J Clin Invest 2008;118:3522-3530.

11 Xiao X, Gaffar I, Guo P, Wiersch J, Fischbach S, Peirish L, Song Z, El-Gohary Y, Prasadan K, Shiota C, Gittes GK: M2 macrophages promote beta-cell proliferation by up-regulation of smad7. Proc Natl Acad Sci U S A 2014;111:E1211-1220.

12 Mills CD: M1 and m2 macrophages: Oracles of health and disease. Crit Rev Immunol 2012;32:463-488.

13 Mills CD, Kincaid K, Alt JM, Heilman MJ, Hill AM: M-1/m-2 macrophages and the th1/th2 paradigm. J Immunol 2000;164:6166-6173.

14 Claassen E, Van Rooijen N: The effect of elimination of macrophages on the tissue distribution of liposomes containing [3h]methotrexate. Biochim Biophys Acta 1984;802:428-434. 


\section{Cellular Physiology Cell Physiol Biochem 2015;37:1279-1288 \begin{tabular}{ll|l} 
and Biochemistry & $\begin{array}{l}\text { DOI 10.1159/000430251 } \\
\text { Published online: October 05, 2015 }\end{array}$ & $\begin{array}{l}\text { O 2015 S. Karger AG, Basel } \\
\text { www.karger.com/cpb }\end{array}$ \\
\cline { 2 - 3 }
\end{tabular} \\ Li et al.: Macrophage Polarization in SLE}

15 van Rooijen N, van Nieuwmegen R: Elimination of phagocytic cells in the spleen after intravenous injection of liposome-encapsulated dichloromethylene diphosphonate. An enzyme-histochemical study. Cell Tissue Res 1984;238:355-358.

16 Plosker GL, Goa KL: Clodronate. A review of its pharmacological properties and therapeutic efficacy in resorptive bone disease. Drugs 1994;47:945-982.

17 Atkins CD: Clodronate in metastatic breast cancer. N Engl J Med 1998;339:1941.

18 Diel IJ, Solomayer EF, Costa SD, Gollan C, Goerner R, Wallwiener D, Kaufmann M, Bastert G: Reduction in new metastases in breast cancer with adjuvant clodronate treatment. N Engl J Med 1998;339:357-363.

19 Meli M, Rausa L: Clodronate in metastatic breast cancer. N Engl J Med 1998;339:1940; author reply 1941.

20 Panasci LC, Sandor V, Melnychuk D: Clodronate in metastatic breast cancer. N Engl J Med 1998;339:19401941; author reply 1941.

21 Saad F: Should patients with prostate cancer without bone metastases receive sodium clodronate? Nature clinical practice Urology 2007;4:584-585.

22 Richards PJ, Williams AS, Goodfellow RM, Williams BD: Liposomal clodronate eliminates synovial macrophages, reduces inflammation and ameliorates joint destruction in antigen-induced arthritis. Rheumatology (Oxford) 1999;38:818-825.

23 Pan B, Liu G, Jiang Z, Zheng D: Regulation of renal fibrosis by macrophage polarization. Cell Physiol Biochem 2015;35:1062-1069.

24 Shen B, Liu X, Fan Y, Qiu J: Macrophages regulate renal fibrosis through modulating TGFbeta superfamily signaling. Inflammation 2014;37:2076-2084.

25 Zhang W, Xu W, Xiong S: Macrophage differentiation and polarization via phosphatidylinositol 3-kinase/ akt-erk signaling pathway conferred by serum amyloid p component. J Immunol 2011;187:1764-1777.

26 Wen ZK, Xu W, Xu L, Cao QH, Wang Y, Chu YW, Xiong SD: DNA hypomethylation is crucial for apoptotic DNA to induce systemic lupus erythematosus-like autoimmune disease in sle-non-susceptible mice. Rheumatology (Oxford) 2007;46:1796-1803.

27 Qiao B, Wu J, Chu YW, Wang Y, Wang DP, Wu HS, Xiong SD: Induction of systemic lupus erythematosus-like syndrome in syngeneic mice by immunization with activated lymphocyte-derived DNA. Rheumatology (Oxford) 2005;44:1108-1114.

28 Weischenfeldt J, Porse B: Bone marrow-derived macrophages (BMM): Isolation and applications. CSH Protocols 2008;2008:pdb prot5080.

29 Davis BK: Isolation, culture, and functional evaluation of bone marrow-derived macrophages. Methods Mol Biol 2013;1031:27-35. 